\title{
Edgebreaker on a Corner Table: A simple technique for representing and compressing triangulated surfaces
}

\author{
Jarek Rossignac, Alla Safonova, Andrzej Szymczak \\ College of Computing and GVU Center \\ Georgia Institute of Technology
}

\begin{abstract}
A triangulated surface $S$ with $V$ vertices is sometimes stored as a list of $T$ independent triangles, each described by the 3 floating-point coordinates of its vertices. This representation requires about 576V bits and provides no explicit information regarding the adjacency between neighboring triangles or vertices. A variety of boundary-graph data structures may be derived from such a representation in order to make explicit the various adjacency and incidence relations between triangles, edges, and vertices. These relations are stored to accelerate algorithms that visit the surface in a systematic manner and access the neighbors of each vertex or triangle. Instead of these complex data structures, we advocate a simple Corner Table, which explicitly represents the triangle/vertex incidence and the triangle/triangle adjacency of any manifold or pseudo-manifold triangle mesh, as two tables of integers. The Corner Table requires about $12 \mathrm{Vlog}_{2} \mathrm{~V}$ bits and must be accompanied by a vertex table, which requires $96 \mathrm{~V}$ bits, if Floats are used. The Corner Table may be derived from the list of independent triangles. For meshes homeomorphic to a sphere, it may be compressed to less that $4 \mathrm{~V}$ bits by storing the "clers" sequence of triangle-labels from the set $\{C, L, E, R, S\}$. Further compression to $3.6 \mathrm{~V}$ bits may be guaranteed by using context-based codes for the clers symbols. Entropy codes reduce the storage for large meshes to less than $2 \mathrm{~V}$ bits. Meshes with more complex topologies may require $\mathrm{O}\left(\log _{2} V\right)$ additional bits per handle or hole. We present here a publicly available, simple, state-machine implementation of the Edgebreaker compression, which traverses the corner table, computes the CLERS symbols, and constructs an ordered list of vertex references. Vertices are encoded, in the order in which they appear on the list, as corrective displacements between their predicted and actual locations. Quantizing vertex coordinates to 12 bits and predicting each vertex as a linear combinations of its previously encoded neighbors leads to short displacements, for which entropy codes drop the total vertex location storage for heavily sampled typical meshes below $16 \mathrm{~V}$ bits.
\end{abstract}

\section{Introdction}

3D graphics plays an increasingly important role in applications where 3D models are accessed through the Internet. Due to improved design and model acquisition tools, to the wider acceptance of this technology, and to the need for higher accuracy, the number and complexity of these models are growing more rapidly than phone and network bandwidth. Consequently, it is imperative to continue increasing the terseness of 3D data transmission formats and the performance and reliability of the associated compression and decompression algorithms.

Although many representations have been proposed for 3D models, polygon and triangle meshes are the de facto standard for exchanging and viewing 3D models. A triangle mesh may be represented by its vertex data and by its connectivity. Vertex data comprises coordinates of all the vertices and optionally the vertex colors and the coordinates of the associated normal vectors and textures. In its simplest form, connectivity captures the incidence relation between the triangles of the mesh and their bounding vertices. It may be represented by a triangle-vertex incidence table, which associates with each triangle the references to its three bounding vertices.

In practice, the number of triangles is roughly twice the number of vertices. Consequently, when pointers or integer indices are used as vertex-references and when floating point coordinates are used to encode vertex locations, uncompressed connectivity data consumes twice more storage than vertex coordinates.

Vertex coordinates may be compressed through various forms of vector quantization. Most vertex compression approaches exploit the coherence in vertex locations by using local or global predictors to encode corrections instead of absolute vertex data. Both the encoder and the decoder use the same prediction formula. The encoder transmits the difference between the predicted and the correct vertex data. It uses variable length codes for the corrections. The better the prediction-the shorter the codes. The decoder receives the correction, decodes it and adds it to the predicted to obtain the correct information for the next vertex. Thus the prediction can only exploit data that has been previously received and decoded. Most predictive schemes require only local connectivity between the next vertex and its previously decoded neighbors. 
Some global predictors require having the connectivity of the entire mesh. Thus it is imperative to optimize connectivity compression techniques that are independent of vertex data.

The Edgebreaker compression scheme, discussed here, has been extended to manifold meshes with handles and holes [Ross99], to triangulated boundaries of non-manifold solids [RoCa99], and to meshes that contain only quadrilaterals or a combination of simply-connected polygonal faces with an arbitrary number of sides [King99b]. It was also optimized for meshes with nearly regular connectivity [SKR00, SKR00b]. Nevertheless, for sake of simplicity, in this chapter, we restrict our focus to meshes that are each homeomorphic to a sphere.

As several other compression schemes [TaRo98, ToGo98, Gust98], Edgebreaker visits the triangles in a spiraling (depthfirst) triangle-spanning-tree order and generates a string of descriptors, one per triangle, which indicate how the mesh can be rebuilt by attaching new triangles to previously reconstructed ones. The popularity of Edgebreaker lies in the fact that all descriptors are symbols from the set $\{\mathrm{C}, \mathrm{L}, \mathrm{E}, \mathrm{R}, \mathrm{S}\}$. No other parameter is needed. Because half of the descriptors are Cs, a trivial code $(\mathrm{C}=0, \mathrm{~L}=110, \mathrm{E}=111, \mathrm{R}=101, \mathrm{~S}=100)$ guarantees that storage will not exceed 2 bits per triangle. A slightly more complex code guarantees 1.73 bits per triangle [King99]. This upper bound on storage does not rely on statistic-based entropy or arithmetic coding schemes, which in general perform poorly on small or irregular meshes. Consequently, the simple encoding of Edgebreaker is particularly attractive for large catalogs of small models and for crude simplifications of complex meshes. For large meshes, entropy codes may be used to further reduce the storage to less than a bit per triangle [RoSz99]. The string of descriptors produced by Edgebreaker is called the clers string. An efficient decompression algorithm for the clers sequence [RoSz99] interprets the symbols to build a simply connected triangulated polygon, which represents the triangle-spanning tree. Then, it zips up the borders of that polygon by matching pairs of its bounding edges in a bottom-up order with respect to the vertex-spanning-tree that is the dual of the triangle-spanning-tree. We describe here a compact implementation of this decompression. A previously proposed alternative, called Spirale Reversi [IsSo99], interprets the reversed clers string and builds the triangle tree from the leaves.

The contributions of this chapter are a simple data structure, called the Corner-Table, for representing the connectivity of triangle meshes and a very compact (single page) description of the complete Edgebreaker compression and decompression algorithms, which trivializes their implementation. The data structure, examples, and source codes for this implementation are publicly available [EB01]. Because the corner table is nothing more than two arrays of integers and because the decompression is simple and fast, the scheme may be suitable for hardware implementation. We first define our notation and introduce the Corner-Table, then we present the simplified Compression and Decompression algorithms.

\section{Notation and Corner-Table}

Vertices are identified using positive integers. Their locations are stored in an array called G for "geometry". Each entry of $\mathrm{G}$ is a 3D point that encodes the location of a vertex. (Other vertex attributes are ignored for simplicity in this chapter.) We have overloaded the "+" and "-" operators to perform additions and subtraction of points and vectors. Thus G[1]-G[0] returns the vector from the first vertex to the second. Edgebreaker compression stores a point and a sequence of corrective vectors in the string called delta, using $\operatorname{WRITE}(d e l t a, \mathrm{D})$ statements, where D is a point or vector. The corrective vectors will be encoded using a variable length binary format in a separate post-processing entropy-compression step. During decompression, the first call READ (delta) returns a decoded version of the first vertex. Subsequent calls to READ (delta) return corrective vectors, which are added to the vertex estimates to produce correct vertices.

Compression stores, in a string called clers, a sequence of symbols from the set $\{\mathrm{C}, \mathrm{L}, \mathrm{E}, \mathrm{R}, \mathrm{S}\}$, encoded using a simple binary format: $\{0,110,111,101,100\}$. Better codes may be substituted easily, if desired.

During decompression, the symbols (i.e., their binary format) are read and used to switch to the correct operation. We assume that the READ instruction knows to read two more bits when the first one is a 1.

The Corner Table data structure used by Edgebreaker is composed of two global arrays (the $\mathrm{V}$ and $\mathrm{O}$ tables) and of two temporary tables $(\mathrm{M}, \mathrm{U})$, which are only used during compression. $\mathrm{V}, \mathrm{O}$, and $\mathrm{U}$ have 3 times as many entries as there are triangles. $\mathrm{M}$ has as many entries as vertices. $\mathrm{V}$ and $\mathrm{O}$ hold the integer references to vertices and to opposite corners. $\mathrm{M}$ and $\mathrm{U}$ hold binary flags indicating whether the corresponding vertex or triangle has already been visited.

Although Edgebreaker manipulates integer indices, we use (our own) object-oriented notation to increase the readability of the algorithms that follow. We use lower-case letters that follow a period to refer to table entries or functions with the corresponding uppercase name. For example, if $\mathrm{c}$ is an integer, c.v stands for $\mathrm{V}[\mathrm{c}]$ and c.o stands for $\mathrm{O}[\mathrm{c}]$. However, when we assign values to specific entries in these tables, we still write $V[c]=b$, rather than $c . v=b$, to remind the reader that we are updating an entry in the $\mathrm{V}$ table. We use left-to-right expansion of this "object-oriented" notation, thus c.o.v stands for $\mathrm{V}[\mathrm{O}[\mathrm{c}]]$.

We also introduce the "next corner around triangle" functions: $N(c)$, which will be written c.n and which returns c-2, if c MOD 3 is 2, and $c+1$ otherwise. This functions permits to move from one corner of a triangle to the next according to the agreed-upon orientation of the triangle, which we assume to be consistent throughout the mesh. The "previous corner 
around triangle" function, written as c.p stands for $\mathrm{N}(\mathrm{N}(\mathrm{c}))$. For example, the statement V[a.p]=b.n.v translates to $\mathrm{V}[\mathrm{N}(\mathrm{N}(\mathrm{a}))]=\mathrm{V}[\mathrm{N}(\mathrm{b})]$.

A corner $\mathrm{c}$ is the association of a triangle c.t with one of its bounding vertices c.v (see Fig.1).. The entries in $\mathrm{V}$ and $\mathrm{O}$ are consecutive for the 3 corners (c.p, c, c.n) of each triangle. Thus, c.t returns the integer division of c by 3 and the cornertriangle relation needs not be stored explicitly. For example, when c is 4, c.t is 1 and thus c is a corner of the second triangle. We use c.t only to mark previously visited triangles in the U table.

The notation c.v returns the id of the vertex associated with corner $\mathrm{c}$. We use this id to mark previously visited vertices in the $\mathrm{M}$ table or to access the geometry of the vertex (c.v.g). The notation c.o returns the id of the corner opposite to c. To be precise, c.o is the only integer $b$ for which: c.n.v == b.p.v and c.p.v == b.n.v. For convenience, we also define c.l as c.p.o and c.r as c.n.o. These relations are illustrated in the figure below. We assume a counter-clockwise orientation.

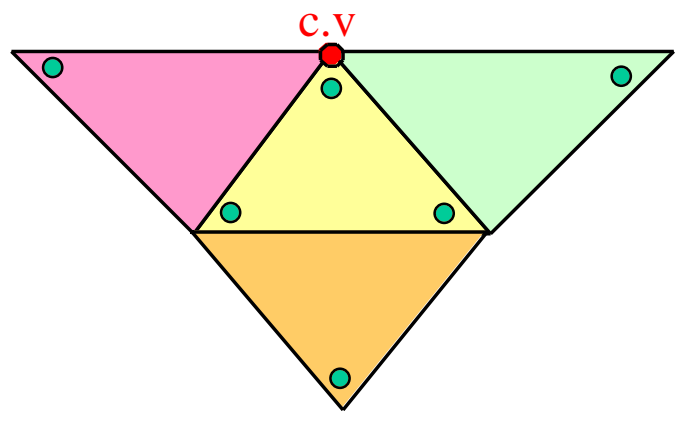

Fig 1: Using the $V$ and $O$ tables, given a corner, $c$, we can access: its triangle, c.t; its vertex, c.v; the previous and next corners, c.p and c.n, in c.t; the opposite corner, c.o; and the corners of the left and right neighbors, c.l and c.r.

\section{Compression}

Edgebreaker is a state machine. At each state it moves from a triangle $\mathrm{Y}$ to an adjacent triangle $\mathrm{X}$. It marks all visited triangles and their bounding vertices. Let Left and Right denote the other two triangles that are incident upon X. Let $\mathbf{v}$ be the vertex common to X, Left, and Right. If $\mathbf{v}$ has not yet been visited, then neither have Left and Right. This is case C. If $\mathbf{v}$ has been visited, we distinguish four other cases, which corresponds to four situations where one, both, or neither of the Left and Right triangles have been visited. These situations and the associated clers symbols are shown in Fig. 2. The arrow indicates the direction to the next triangle. Previously visited triangles are not shown. Note that in the $\mathrm{S}$ case, Edgebreaker moves to the right, using a recursive call, and then to the left.

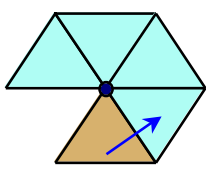

$\mathbf{L}$

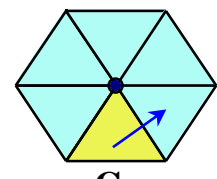

C

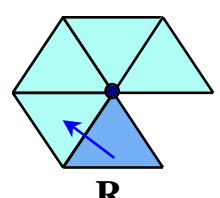

$\mathbf{R}$

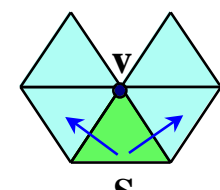

$\mathbf{S}$

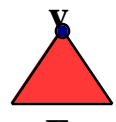

$\mathbf{E}$

Fig 2: Using the $V$ and $O$ tables, given a corner, $c$, we can access: its triangle, c.t; its vertex, c.v; the previous and next corners, c.p and c.n, in c.t; the opposite corner, c.o; and the corners of the left and right neighbors, c.l and c.r.

The compression algorithm is composed of an initialization followed by a call to Compress. The initial corner $c$ may be chosen randomly. The initialization encodes and marks the three vertices of the first triangle, marks the triangle as visited, and calls compress.

Compress is a recursive procedure that traverses the mesh along a spiraling triangle-spanning tree. A recursion starts at triangles that are of type S. It compresses the branch adjacent to the right edge of such a triangle. When the corresponding E triangle is reached, the branch traversal is complete and we "RETURN" from the recursion to pursue the left branch. The encounter of an $\mathrm{E}$ that does not match an $\mathrm{S}$ terminates the compression process. If the tip vertex of a new triangles has not yet been visited ("IF c.v.m != 1"), we are on a C triangle and we encode in delta the corrective vector for the tip of the current triangle using a parallelogram rule [ToGo98]. We also encode a C symbol (for example code(C) may return a 0 ) in the clers string. When the tip of the new triangle has been visited, we distinguish amongst the four other cases, based on the status of the neighboring (left and right) triangles. The compression pseudo-code is provided in the frame below. 


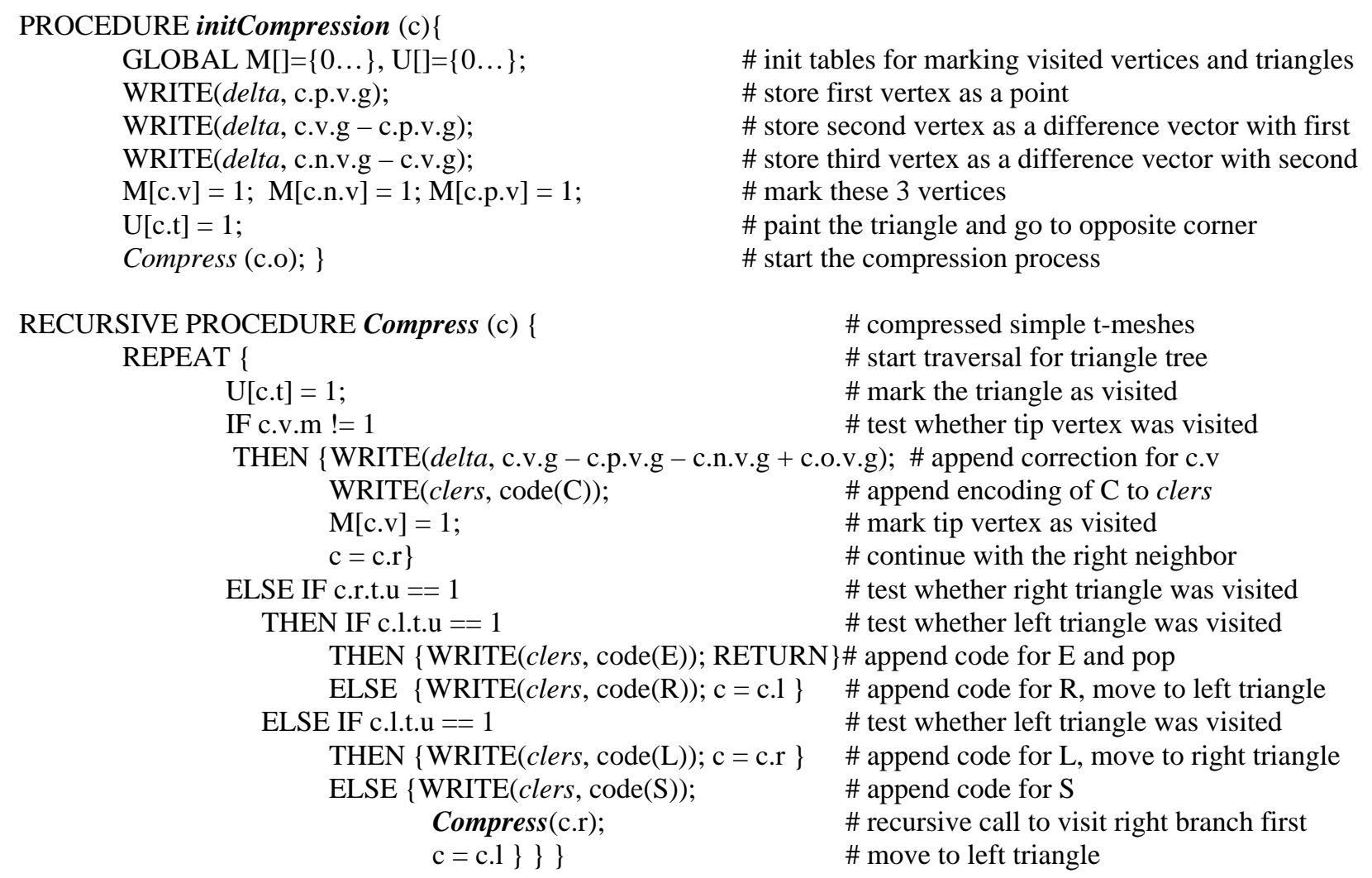

The Fig.3, below, shows the labels for triangles that have been visited during a typical initial phase of compression.

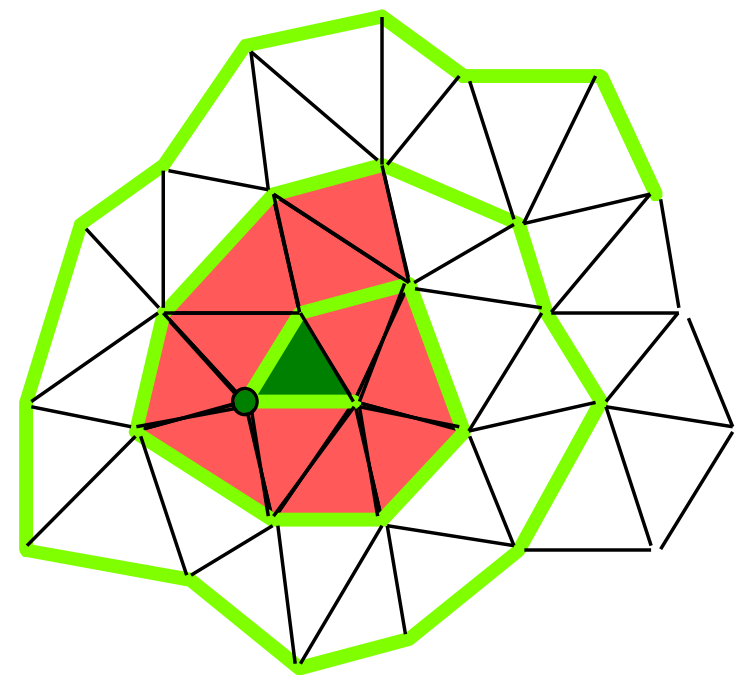

Fig 3: The clers sequence CCCCRCCRCRC is produced as Edgebreaker starts compressing this mesh.

Fig. 4 shows the final steps of compression for a branch or for the whole mesh. It appends the symbols CRSRLECRRRLE to clers. The first triangle is marked by an arrow. 


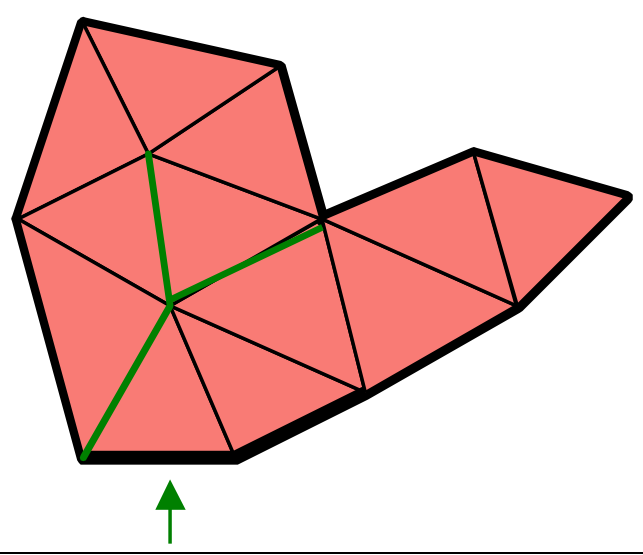

Fig 4: The clers sequence CRSRLECRRRLE is produced as Edgebreaker finishes compressing this mesh.

\section{Decompression}

The decompression algorithm builds the two arrays, $\mathrm{V}$ and $\mathrm{O}$, of the corner Corner-Table and the $\mathrm{G}$ table of vertex locations. After initializing the first triangle in initDecompression, the recursive procedure Decompress is called for corner 1. In each iteration, Edgebreaker appends a new triangle to a previously visited one. It interprets the binary encoding of the next symbol from the clers string. If it is a $\mathrm{C}$, Edgebreaker associates the label -1 with the corner opposite the left edge and stores if in $\mathrm{O}$. This temporary marking will be replaced with the correct reference to the opposite corner by a zip. If the symbol is an L, Edgebreaker associates a different label $(-2)$ with the opposite edge and tries to zip, by identifying it with the adjacent edge on the left. When an $\mathrm{R}$ symbol is encountered, the opposite edge is labeled -2 . No zipping takes place. When an E symbol is encountered, both edges are labeled -2 , and an iterative zipping is attempted. This zipping will continue as long as the free edge on the right of the last zipped vertex is marked with -2 and the free edge on the left is marked -1. An S symbols forks a recursive call to Decompress, which will construct and zip a subset of the mesh that is incident to the right edge of the current triangle. Then the reconstruction proceeds to decode and build the branch attached to the left edge of the current triangle. Typically about $2 \%$ of the triangles are of type S. The process is illustrated in Fig. 5.

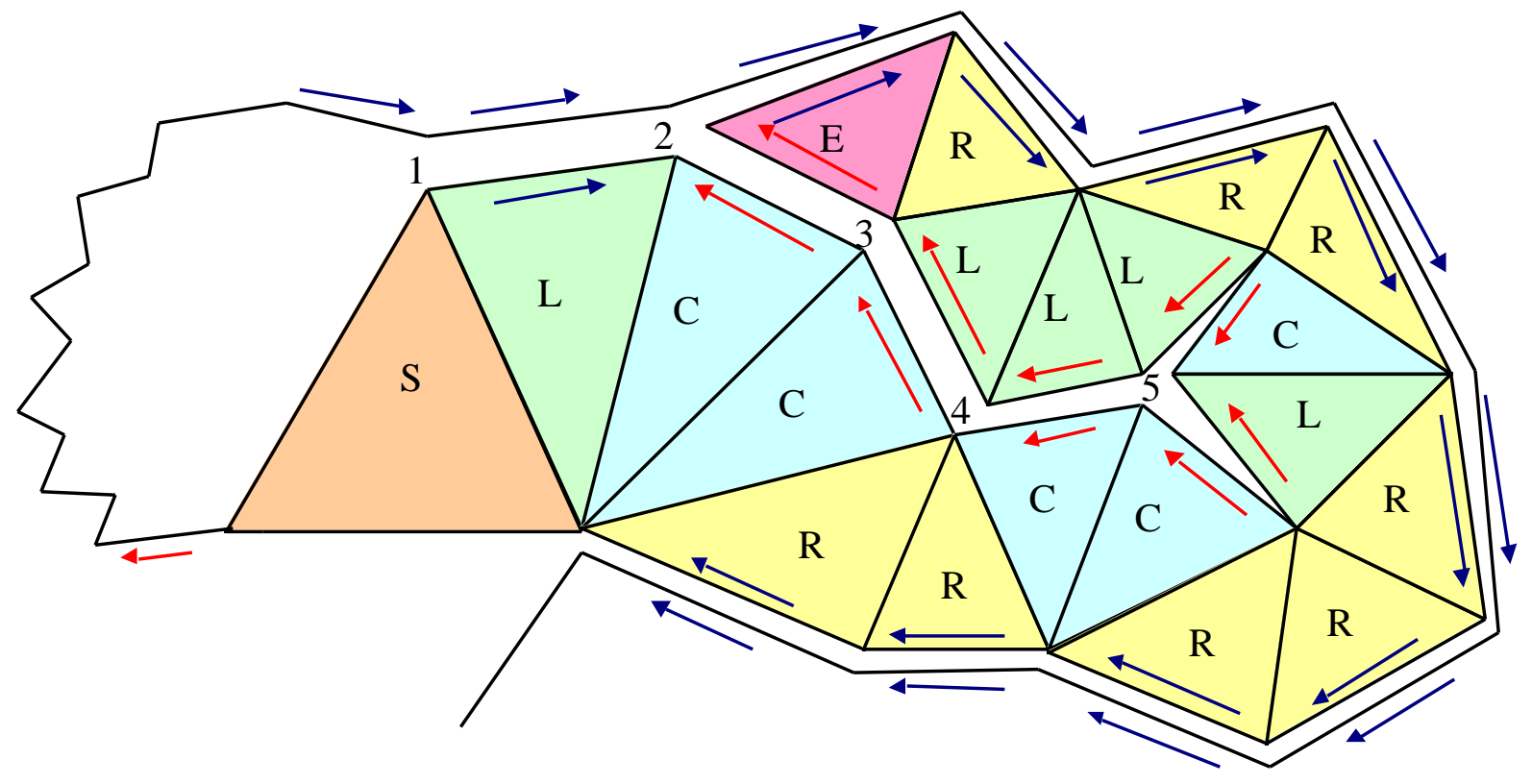

Fig 5: The clers string SLCCRRCCRRRLCRRLLLRE will generate the mesh shown here. The left edge of the first $L$ triangle is not zipped immediately. The left edge of the second $L$ triangle is zipped reaching vertex 5. Then, as we encounter the subsequent three L triangles, their left edges are zipped right away. The first left edge of the E triangle is also zipped. The rest will be zipped later, when the left branch of the S triangle is decoded.. 


\begin{tabular}{|c|c|}
\hline \multicolumn{2}{|l|}{ PROCEDURE initDecompression \{} \\
\hline GLOBAL V []$=\{0,1,2,0,0,0,0,0, \ldots\}$ & \# table of vertex Ids for each corner \\
\hline GLOBAL O $]=\{-1,-3,-1,-3,-3,-3 \ldots\}$ & \# table of opposite corner Ids for each corner \\
\hline GLOBAL T = 0 & \# id of the last triangle decompressed so far \\
\hline GLOBAL N = 2; & \# id of the last vertex encountered \\
\hline DecompressConnectivity(1); & \# starts connectivity decompression \\
\hline GLOBAL $\mathrm{M}[]=\{0 \ldots\}, \mathrm{U}[]=\{0 \ldots\}$ & les for marking visited vertices and triangles \\
\hline $\mathrm{G}[0]=\mathrm{READ}($ delta $)$ & \# read first vertex \\
\hline $\mathrm{G}[1]=\mathrm{G}[0]+\mathrm{READ}($ delta $)$ & \# set second vertex using first plus delta \\
\hline $\mathrm{G}[2]=\mathrm{G}[1]+\mathrm{READ}($ delta $)$ & \# set third vertex using second plus new delta \\
\hline GLOBAL $\mathrm{N}=2$ & \# id of the last vertex encountered \\
\hline $\mathrm{M}[0]=1 ; \mathrm{M}[1]=1 ; \mathrm{M}[2]=1$ & \# mark these 3 vertices \\
\hline $\mathrm{U}[0]=1$ & \# paint the triangle and go to opposite corner \\
\hline DecompressVertices $(\mathrm{O}[1]) ;\}$ & \# starts vertices decompression \\
\hline \multicolumn{2}{|l|}{ RECURSIVE PROCEDURE DecompressConnectivity (c) \{ } \\
\hline REPEAT \{ & \# Loop builds triangle tree and zips it up \\
\hline $\mathrm{T}++$ & \# new triangle \\
\hline $\mathrm{O}[\mathrm{c}]=3 \mathrm{~T} ; \mathrm{O}[3 \mathrm{~T}]=\mathrm{c}$ & \# attach new triangle, link opposite corners \\
\hline $\mathrm{V}[3 \mathrm{~T}+1]=\mathrm{c} \cdot \mathrm{p} \cdot \mathrm{v} ; \mathrm{V}[3 \mathrm{~T}+2]=\mathrm{c} \cdot \mathrm{n} \cdot \mathrm{v}$ & \# enter vertex Ids for shared vertices \\
\hline $\mathrm{c}=\mathrm{c} . \mathrm{o} . \mathrm{n}$ & \# move corner to new triangle \\
\hline Switch decode $(\operatorname{READ}($ clers $))\{$ & \# select operation based on next symbol \\
\hline Case $\mathrm{C}:\{\mathrm{O}[\mathrm{c} . \mathrm{n}]=-1 ; \mathrm{V}[3 \mathrm{~T}]=++\mathrm{N} ;\}$ & \# C: left edge is free, store ref to new vertex \\
\hline Case L: $\{\mathrm{O}[\mathrm{c} . \mathrm{n}]=-2 ; z i p(\mathrm{c} . \mathrm{n}) ;\} \quad \# \mathrm{~L}$ & ent free edge, try to zip once \\
\hline Case R: $\{\mathrm{O}[\mathrm{c}]=-2 ; \mathrm{c}=\mathrm{c} . \mathrm{n}\}$ & \# R: orient free edge, go left \\
\hline Case S: \{ DecompressConnectivity(c); & c.n \} \# S: recursion going right, then go left \\
\hline Case E: $\{\mathrm{O}[\mathrm{c}]=-2 ; \mathrm{O}[\mathrm{c} . \mathrm{n}]=-2 ; z i p($ & RETURN \}\}\}\} \# E: zip, try more, pop \\
\hline & \# tries to zip free edges opposite c \\
\hline $\begin{array}{l}\text { RECURSIVE PROCEDURE Zip (c) }\{ \\
\qquad \mathrm{b}=\text { c.n; WHILE b.o }>=0 \text { DO b=b.o.n; }\end{array}$ & \# search clockwise for free edge \\
\hline & \# pop if no zip possible \\
\hline $\begin{array}{l}\text { IF b.o } !=-1 \text { THEN RETURN; } \\
\mathrm{O}[\mathrm{c}]=\mathrm{b} ; \mathrm{O}[\mathrm{b}]=\mathrm{c} ;\end{array}$ & \# link opposite corners \\
\hline $\mathrm{a}=\mathrm{c} . \mathrm{p} ; \mathrm{V}[\mathrm{a} \cdot \mathrm{p}]=$ b.p.v; & \# assign co-incident corners \\
\hline \\
\hline $\begin{array}{l}\text { WHILE a.o }>=0 \& \& \text { b!=a DO }\{\text { a=a.o.p; V[a.p]=b.p.v }\} \\
\text { c = c.p; WHILE c.o }>=0 \& \& \text { c!= b DO c = c.o.p; }\end{array}$ & $\begin{array}{l}\text { \# find corner of next free edge on right } \\
\text { \# try to zip again }\end{array}$ \\
\hline \multicolumn{2}{|l|}{ RECURSIVE PROCEDURE DecompressVertices(c) \{ } \\
\hline REPEAT \{ & \# start traversal for triangle tree \\
\hline $\mathrm{U}[\mathrm{c} . \mathrm{t}]=1$ & \# mark the triangle as visited \\
\hline IF c.v.m != 1 & \# test whether tip vertex was visited \\
\hline \multicolumn{2}{|c|}{ THEN $\{\mathrm{G}[++\mathrm{N}]=$ c.p.v.g+c.n.v.g-c.o.v.g+READ(delta); \# update new vertex } \\
\hline \multirow{2}{*}{$\begin{array}{l}\mathrm{M}[\mathrm{c} . \mathrm{v}]=1 \\
\mathrm{c}=\mathrm{c} . \mathrm{r} ;\}\end{array}$} & \# mark tip vertex as visited \\
\hline & \# continue with the right neighbor \\
\hline ELSE IF c.r.t.u $==1$ & \# test whether right triangle was visited \\
\hline THEN IF c.l.t.u == 1 & \# test whether left triangle was visited \\
\hline THEN RETURN & \# рор \\
\hline & \# move to left triangle \\
\hline & \# test whether left triangle was visited \\
\hline $\begin{array}{l}\text { ELSE IF c.l.t.u }==1 \\
\text { THEN }\{\mathrm{c}=\mathrm{c} . \mathrm{r}\}\end{array}$ & \# move to right triangle \\
\hline $\begin{array}{c}\text { ELSE }\left\{\begin{array}{c}\text { DecompressVertices }(c . r) \\
c=c .1\}\end{array}\right\}\end{array}$ & $\begin{array}{l}\text { \# recursive call to visit right branch first } \\
\text { \# move to left triangle }\end{array}$ \\
\hline
\end{tabular}

The pseudocode for the decompression algorithm is shown in the frame above. 


\section{Conclusion}

3D mesh compression and planar graph encoding techniques have been the subject of numerous publications (see [Ross99] for a review of prior art). All these approaches have been presented at a high level. Many are complex and difficult to implement. In comparison, the proposed compression and decompression algorithms are trivial to implement. More importantly, the source code is extremely small and uses simple arrays of integers as a data structure. This simplicity makes them suitable for many Internet and possibly even hardware applications.

\section{Acknowledgements}

This material is based upon work supported by the National Science Foundation under Grant 9721358.

\section{References}

[GuSt98] S. Gumhold and W. Strasser, "Real Time Compression of Triangle Mesh Connectivity", Proc. ACM Siggraph, pp. 133-140, July 1998.

[IsSo99] M. Isenburg and J. Snoeyink, "Spirale Reversi: Reverse decoding of the Edgebreaker encoding", Tech. Report TR-99-08, Computer Science, UBC, 1999.

[King99] D. King and J. Rossignac, "Guaranteed 3.67V bit encoding of planar triangle graphs", 11th Canadian Conference on Computational Geometry (CCCG"99), pp. 146-149, Vancouver, CA, August 15-18, 1999.

[King99b] D. King and J. Rossignac, "Connectivity Compression for Irregular Quadrilateral Meshes" Research Report GIT-GVU- 99 29, Dec 1999.

[RoCa99] J. Rossignac and D. Cardoze, "Matchmaker: Manifold Breps for non-manifold r-sets", Proceedings of the ACM Symposium on Solid Modeling, pp. 31-41, June 1999.

[Ross99] J. Rossignac, "Edgebreaker: Connectivity compression for triangle meshes", IEEE Transactions on Visualization and Computer Graphics, 5(1), 47-61, Jan-Mar 1999. (Sigma Xi award: Best Paper from Georgia Tech.)

[RoSz99] J. Rossignac and A. Szymczak, "Wrap\&Zip decompression of the connectivity of triangle meshes compressed with Edgebreaker", Computational Geometry, Theory and Applications, 14(1/3), 119-135, November 1999.

[SKR00] A. Szymczak,. D. King, J. Rossignac, "An Edgebreaker-based efficient compression scheme for regular meshes", Proc of the 12th Canadian Conference on Computational Geometry, Fredericton, New Brunswick, August 16-19, 2000.

[SKR00b] A. Szymczak, D. King, J. Rossignac, "An Edgebreaker-based Efficient Compression Scheme for Connectivity of Regular Meshes", Journal of Computational Geometry: Theory and Applications, 2000.

[TaRo98] G. Taubin and J. Rossignac, "Geometric Compression through Topological Surgery", ACM Transactions on Graphics, 17(2), 84-115, April 1998. (IBM award: Best Computer Science Paper from IBM.)

[ToGo98] C. Touma and C. Gotsman, “Triangle Mesh Compression”, Proceedings Graphics Interface 98, pp. 26-34, 1998. 